\title{
Development of a New Ultrasonic Urine Sensor for Bladder Measurement
}

\author{
Hiroyuki Kodama*, Kejin HuanG*, Jing Yu*, \\ Yasuo Kuchinomachi**, Hisashi Yoshimura ${ }^{* * *}$ and Mitsuo WadA ${ }^{* * * *}$
}

\begin{abstract}
A new ultrasonic urine sensor is developed for measuring the urine accumulation in the human bladder. Intensive observations are made on the bladder expansion process under the gradual accumulation of urine by using a magnetic resonance imager. It is observed that the primary changes in shape take place at the top and very small changes occur at the bottom. These findings help to establish a simple and yet reasonable mechanism for the bladder measurement because the variations in the height of the bladder can be easily ascertained with a proposed $\mathrm{PD}$ index in terms of the application of ultrasound beams. The principle of measurement is evaluated in a clinical test and a strong monotonic tendency is identified between the urine accumulation and the proposed PD index. Design of the new ultrasonic urine sensor is then conducted and further experimental tests are carried out with the apparatus developed. It is found that the new ultrasonic urine sensor can really display a very good indication of the urine accumulation in the bladder. The new ultrasonic urine sensor is already commercialized and has received high appraisal from a great number of users. It is characterized by non-invasive measurement, small volume, low weight and affordable price, thereby permitting easy access by various patients including older adults.
\end{abstract}

Key Words : ultrasonic urine sensor, bladder measurement, prediction model, data processing, clinical test, commercialization

\section{Introduction}

Although maintaining independence and active participation in society is something that everyone hopes to attain, such freedom may simply be deprived when one has an annoying disease. Such kinds of diseases may not be so serious in nature; however, they can deteriorate terribly the qualities of daily life and impair considerably the dignity of patients, thereby leading to a desperate feeling and the degradation of social activities. One of the typical diseases is associated with the inability to void the bladder appropriately, namely, urinary incontinence. Such disease can generally be aroused in two alternative ways. One is due to the serious damage of the spinal cord caused by various accidents or diseases. The scarcity of neurogenic

* Department for Human Science and Biomedical Engineering, National Institute of Advanced Industrial Science and Technology (AIST), Tsukuba

** Faculty of Social Welfare System, Shizuoka University of Welfare, Yaizu

*** Takeshiba Engineering Inc., Kanagawa

**** Graduate School of Information Science and Technology, Hokkaido University, Sapporo

(Received March 1, 2006)

(Received August 31, 2006) signals or even no neurogenic signals at all between the brain and the bladder causes the patients impossible to know correctly the state of urine accumulation and thus the appropriate time to void the bladder, leaving this kind of patients frequently at the risk of bladder over-distention. Should no necessary and proper measures be taken, the bladder over-distention can gradually lead to permanent kidney damage and subsequent renal failure. The other is due to the physiological disorder caused mainly by, for instance, the cerebrovascular disease and frequently happens to older adults. Although the reflex from the urine accumulation in the bladder is generally considered normal for these patients, urinary incontinence or urine leakage can not be well prevented because of the low speed of movement (i.e., low mobility) and thus the relatively long time that must be waited before the bladder can be voided. It is roughly estimated that nearly 20 percent of the older adults has, to a various degree, been suffering from this kind of symptoms and no effective medical treatment is available now. Currently, the only way to relieve the stress of urinary incontinence is still the use of indwelling catheters or diapers (i.e., urine receiver). In addition to the drawbacks of labor intensive and cumbersome, the patients themselves have to endure the suffering 
計測自動制御学会論文集

and the risk of infection that may be caused by the nursing methods adopted. Although a great number of countries especially developed ones have already entered into an aged society, there seems to be no essential progress in the development of social welfare and health care system. From now on, it is therefore of great significance to gain steady improvement in the social welfare and health care system so as to maintain the well-being of patients and reduce the labor intensity of nursing workers (to express more exactly, a very big burden in the aged society) as well in the near future.

One of the primary obstacles that preclude mankind from solving the above problems is the difficulties in the accurate detection of the urine accumulation in the bladders of patients. If the information of the urine accumulation can be known in advance by the patients and/or nursing staffs, then proper actions can be taken in time and the efficiency and quality of nursing care can be improved considerably. For instance, urine can be withdrawn at any time when it is necessary for those who have serious ailments in their spinal cords. The risk of bladder overdistention can thus be avoided and it is no longer necessary to take an indwelling catheter 24 hours a day. For older patients, they may have enough time to void their bladders and urinary incontinence or urine leakage might not be a problem at all. As a result, the problems of infection caused by diapers and indwelling catheters could be overcome completely. It should be indicated here that although the measurement of the urine accumulation in the human bladder is so important to the stable life and well-being of various patients annoyed by urinary incontinence, the relevant research and development on the bladder measurement has not been given enough attention and only a few studies have been reported so far. Before 1980, a couple of measurement apparatus were once invented, their accuracy was, however, far from satisfactory. In particular, they were either too expensive to afford or too bulky to wear continuously, thereby preventing them from being used easily by patients with urinary incontinence ${ }^{1), 2}$. In 1986, Companion et al. ${ }^{3)}$ developed an ultrasonic urine sensor, where the intensity of the echoes reflected from the bladder was converted into the prediction of the urine accumulation through an embedded model. Although it was fairly simple in principle, the accurate measurements of urine accumulation in the bladder could sometimes not be guaranteed. Later, Diagnostic Ultrasound Corporation (Seattle of Washington, USA) commercialized a portable three dimensional ultrasonic scanning device with a probe that could sequentially scan the bladder with an increment angle of $15^{\circ 4)}$. Although some researchers showed that it could allow a relatively accurate measurement of the urine accumulation even when the bladder is less than $100 \mathrm{ml}$ in volume, the accuracy of the measurement could be affected seriously by a number of factors from both patients and their doctors ${ }^{5)}$ ). In addition to the relatively complicated measurement principle (thus relatively expensive in price), its performance also depended heavily upon the dynamic behavior of the step motor mounted and the condition of the chargeable battery used. In 1999, Ganguly et al. ${ }^{8)}$ patented a more delicate design of an ultrasonic urine sensor. The bladder was first sectioned by a number of transverse planes that are formed with several groups of ultrasound beams. The bladder volume was then estimated in terms of the angles between the groups of ultrasound beams and the cross sectional area of the bladder on the transverse planes. Although it was said to be relatively accurate for the bladder measurement, it was too complicated in principle and yet too expensive (i.e., too many piezo-electric elements employed) to use in daily life. Therefore, in order to fully meet the needs of various patients annoyed by urinary incontinence, it is imperative to work out a simple, cheap and yet reliable method for the bladder measurement.

In this work we report in great detail the development and design of a new ultrasonic urine sensor. First of all, the expansion characteristics of the bladder under the gradual accumulation of urine are drawn in terms of close observations with a magnetic resonance imager. By means of an intensive clinical evaluation conducted in the Spinal Injuries Center (Iizuka City of Fukuoka Prefecture, Japan) through an effective cooperation with 23 persons including 20 men and 3 women, a much simple principle of bladder measurement is established. Design of the new ultrasonic urine sensor is then carried out and further experimental tests are performed in the Takeshiba Engineering Incorporation (Sagamihara City of Kanagawa Prefecture, Japan) and the National Institute of Advanced Industrial and Science Technology (Tsukuba City of Ibaraki Prefecture, Japan). The performance of the apparatus developed is seriously examined regarding its measurement accuracy and robustness. Potential applications of the new ultrasonic urine sensor are pointed out and future research work is indicated for the further refinement of this device. Some concluding remarks are finally made in the last section of the paper. 


\section{Expansion Characteristics of the Human Blad- der}

It should be indicated here that although the expansion process of the bladder is closely relevant to the measurement of urine accumulation almost no methods proposed so far have taken the advantages of its expansion characteristics. To develop a simple and yet reliable measurement principle, it appears to be beneficial to carefully investigate the expansion process of the bladder under the condition of gradual accumulation of urine and this can be achieved in terms of a magnetic resonance imager (MRI). In Fig. 1, a typical expansion process of the bladder is demonstrated from three (i.e., sagittal, coronal and transverse) directions, respectively. At the beginning of the observations, the person photographed has to void his bladder and lies on his back (i.e., in a supine position) with his abdomen in the center of the magnetic field of the MRI. Photographs are then taken from different directions at 30 minutes, $1 \mathrm{hr}, 1.5 \mathrm{hrs}$ and $2 \mathrm{hrs}$, respectively. Based on Fig. $1 \mathrm{a}$, it is readily understood that the gradual accumulation of urine gives rise to the variations in shape primarily at the top part of the bladder. There appear almost no distinct changes in the other parts of the bladder. In particular, the bottom area of the bladder demonstrates

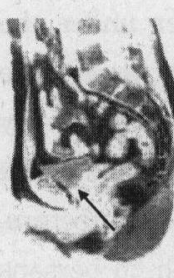

$\mathrm{t}=0.5 \mathrm{hr}$

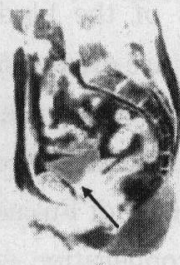

$\mathrm{t}=1 \mathrm{hr}$

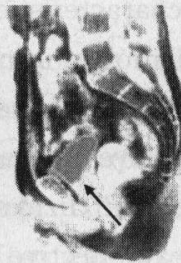

$\mathrm{t}=1.5 \mathrm{hr}$

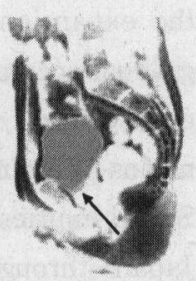

$\mathrm{t}=2 \mathrm{hr}$ (a)

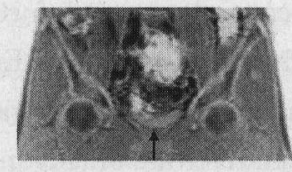

$\mathrm{t}=0.5 \mathrm{hr}$

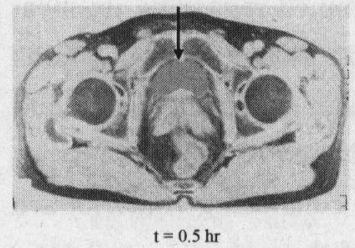

$\mathrm{t}=0.5 \mathrm{hr}$ (b)

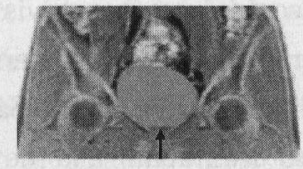

$\mathrm{t}=2 \mathrm{hr}$

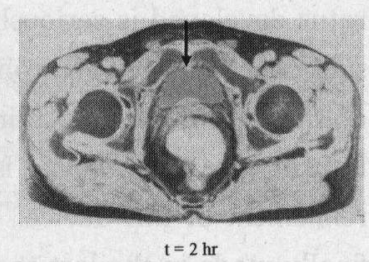

c)
Fig. 1 Expansion of the bladder under the urine accumulation observed by a MRI. (a ) sagittal section; ( b ) coronal section; (c) transverse section very small changes probably due to the existence of pubis and pelvis. In terms of Fig. $1 \mathrm{~b}$ and Fig. 1c, similar observations can also be made. These unique characteristics of the bladder expansion process should be fully used in order to derive a measurement method as simple as possible.

\section{Principle of Bladder Measurement and its Eval- uations}

According to the above observations on the bladder expansion process, it seems to be reasonable to just infer the variations in shape in the top part and ignore the fairly small changes in the other parts. Thus, the urine accumulation in the bladder can, in principle, be simply estimated with its bottom area multiplied by its height. The irregular changes in the shape of the bladder can be taken into account later in the determination of model structures and the parameter estimation with the least-squares regression method. The height of the bladder can be estimated with the application of ultrasonic beams because it has already found wide applications in the diagnosis and examination of internal organs of human being ${ }^{9) \sim 11)}$. To gain a relationship between the height of the bladder and the occurrence of the echoes reflected, the following measurement index has been devised.

$$
P D=\sum_{i=1}^{i=n} P_{i} D_{i}
$$

where $P_{i}$ are the intensities of the echoes reflected from the rear wall of the bladder; $D_{i}$, the distances between the echoes reflected respectively from the back wall and front wall of the bladder and $n$, the number of ultrasonic beams employed.

It should be indicated here that the summation operation in the $P D$ index plays an important role in the estimation of the height of the bladder. If the intensity of an echo reflected is very strong, it indicates that the bladder has expanded to the level where the ultrasonic beam is located. On the contrary, if the intensity of an echo reflected is very weak, it indicates that the bladder has not yet approached the level where the ultrasonic beam is located. The inclusion of $P_{i}(i=1, \cdots, n)$ and $D_{i}(i=1, \cdots, n)$ makes the $P D$ index with some additional features. For instance, because $P_{i}(i=1, \cdots, n)$ and $D_{i}(i=1, \cdots, n)$ contain respectively the information of intensities of the echoes reflected and the shape of the bladder, they both help to reflect the real status of the bladder measured.

In order to evaluate the devised principle of bladder measurement, a clinical test is needed to be carried out and the test plan is approved by the administration office of the Spinal Injuries Center. Simultaneous examination of 
cystometry (i.e., flow dynamics and its relationship with the pressure in the bladder) and measurement of the amount of physiological saline water injected into the bladder are carried out in close cooperation with 23 persons including 20 men and 3 women. They aged from 14 to 74 years-old, covering approximately the lifespan of human being. They all suffer, by a certain extent, from the injury of their spines and full written consent has been received before the detailed clinical test. The relationship between the amount of physiological saline water injected into the bladders and the corresponding measurement indexes is satisfactorily obtained. Based on the characteristics of the relationship, the results obtained can be further categorized into four subgroups as shown in Fig. 2. For the first subgroup of 6 persons (Fig. 2a), the measurement index takes an extremely good linear relationship with the amount of physiological saline water injected into the bladders. For the second subgroup of 8 persons, the linear relationship can still be observed despite the fact that the degree of linearity becomes somewhat worse than in the first subgroup (Fig. 2b). For the third subgroup of 6 persons, it appears to be difficult to observe a linear relationship between the amount of physiological saline water injected and the measurement index (Fig. 2c). Nevertheless, a monotonic tendency can still be identified clearly if the physiological saline water injected has been considered in two separate regions: a low volume region $(V<100 \mathrm{ml})$ and a high volume one $(V \leq 100 \mathrm{ml})$. It indicates that the nonlinearity of the bladder expansion processes has to be considered in this subgroup. The worst circumstance occurs in the fourth subgroup of 3 persons and almost no corresponding relationship can be found

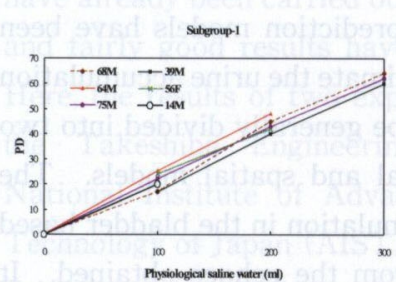

(a)

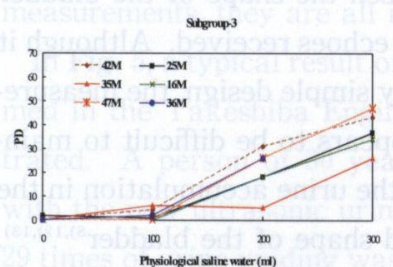

(c)

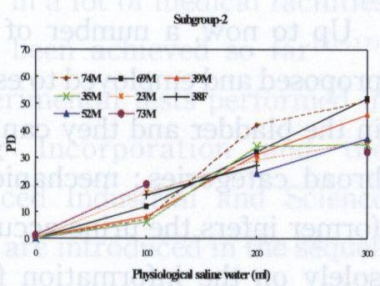

(b)

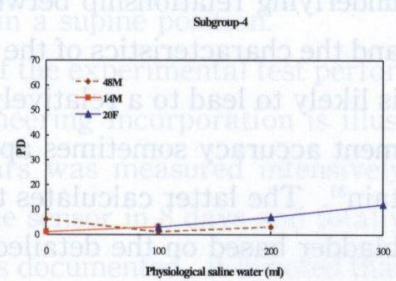

(d)
Fig. 2 A clinical test conducted in the Spinal Injuries Center of Japan actually (Fig. 2d). Further studies are then carried out for this subgroup in terms of $\mathrm{x}^{-}$-ray examination (In fact, $\mathrm{x}^{-}$ ray examination is conducted for all subgroups and no particular symptoms are found in the first three subgroups). It is found that the irregular shape and the location of the bladders causes the bladders to be no longer in the normal measurement area of the ultrasonic beams. Adjustment of the locations of the ultrasonic beams is tried and a monotonic tendency is reproduced. The severe change in the shape of the bladder is frequently aroused by the bladder complications peculiar to the serious injuries of the spinal cord.

The outcome of the clinical test looks very striking because a definite monotonic relationship between the urine accumulation in the bladder and the corresponding measurement index has been identified for almost all the persons tested. Based on these encouraging results, it seems reasonable to reach a conclusion that the measurement index devised can really reflect the expansion process of the bladder incurred by the accumulation of urine.

\section{Development of the New Ultrasonic Urine Sen-} sor

The new ultrasonic urine sensor is developed in accordance with the above measurement principle. A simplified functional block diagram of the new ultrasonic urine sensor is shown in Fig. 3a. As can be seen, it consists of a transducer, a digitizer and a microprocessor. The transducer sends ultrasonic beams ( $f=3 \mathrm{MHz}$ ) to the bladder and also receives the echoes reflected. Four piezo-electric elements (represented respectively by $a, b, c$ and $d$ hereinafter) are in general considered to be enough for the

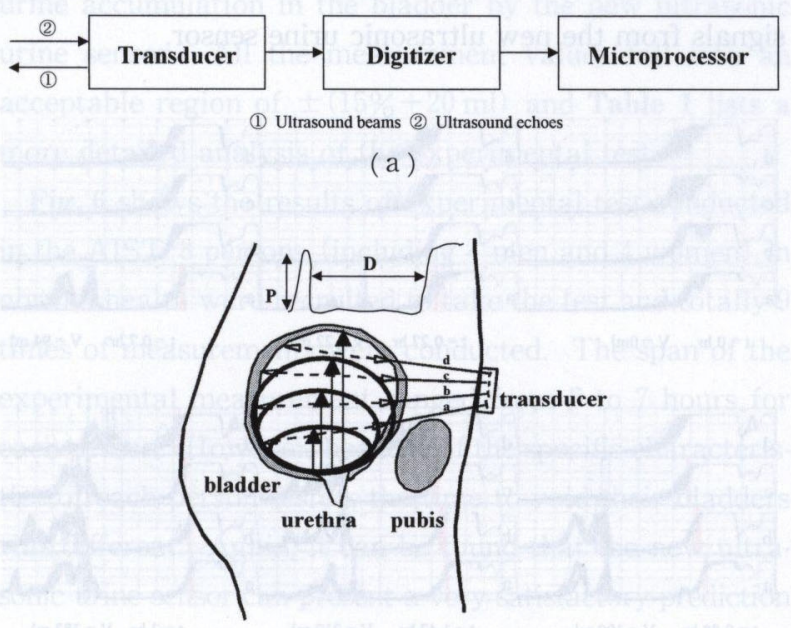

(b)

Fig. 3 Design of the new ultrasonic urine sensor. (a functional block diagram; (b) measurement principle 
coverage of urine accumulation in the bladder $(0 \leq V \leq 900$ $\mathrm{ml}$ ). They are embedded in the transducer and arranged in a line along the cephalic direction with the element a at the bottom and $d$ at the top (c.f., Fig. 3b). The digitizer converts the analog signals from the transducer into the digital ones and finds the appropriate values of $P_{i}$ and $D_{i}$ ( $i=\mathrm{a}, \mathrm{b}, \mathrm{c}, \mathrm{d}$ ). The microprocessor pre-treats the digitized data and then calculates the amount of urine in the bladder. If the urine accumulation thus calculated exceeds a predetermined overflow threshold, then it sends alert signals to the patient. Other devices, for instance, a CRT display and a memory for storing historical data may be connected to the microprocessor.

The design of the new ultrasonic urine sensor is well illustrated in Fig. 3b. During the measurement, the transducer has to be acoustically impedance matched to the skin of the patient in the low abdomen. Upon the accumulation of urine in the bladder, the number of piezo-electric elements that can receive the echoes reflected is increased, thereby augmenting the intensity of the echoes and being able to reflect the enlargement of the bladder with $P D=$ $\sum_{i=a}^{i=d} P_{i} D_{i}$. In Fig. 4, a number of responses of the new ultrasonic urine sensor are displayed when the bladder of a person of 30 years has been filled with different amount of urine at different instants. Here, the red lines stand for the position of the echoes from the front wall of the bladder and the blue lines the position of the echoes from the back wall of the bladder. It is readily seen that the number of piezo-electric elements receiving echoes reflected from the back wall of the bladder increases gradually with the accumulation of urine in the bladder. This exhibits clearly a monotonic relationship between the amount of urine retained in the bladder and the output signals from the new ultrasonic urine sensor.
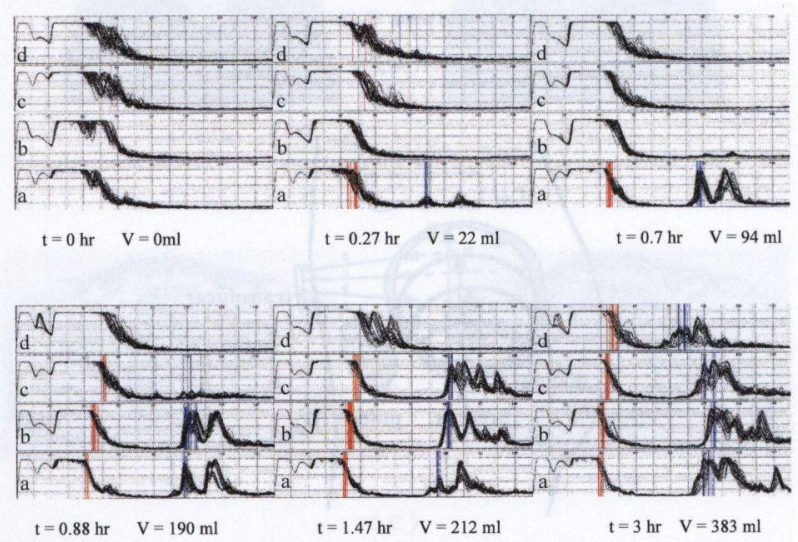

Fig. 4 The number of piezo-electric elements receiving echoes from the bladder versus the condition of urine accumulation (red line: front wall; blue line: back wall)
Unlike those previously developed ultrasonic urine sensors, for instance the one by Ganguly et $\mathrm{al}^{8}{ }^{8}$, the new ultrasonic urine sensor appears to be not so sensitive to the method for determining the distance between the front and the back walls of the bladder, thereby rendering the design of the device relatively easy and improving the robustness of the measurement. In fact, the way to determine the position of the front and the back walls of the bladder can influence seriously the accuracy of the measurement and usually some very complicated searching algorithms including iterative ones have to be adopted. With the application of the new measurement principle, the determination of the front and the back walls of the bladder can be substantially simplified.

\section{Data Pre-Processing and Prediction Models}

It should be pointed out here that the signals reflected from the bladder appear to be very noisy due to probably the inherent characteristics of the ultrasound waves. This kind of phenomena has been found actually in all kinds of measurements for internal organs of human being with the application of ultrasound beams. The fairly zigzag nature of measurement signals prohibits the new ultrasound urine sensor from becoming a reliable and accurate medical instrument and it is therefore necessary to carefully filter out the noise from the digitalized data. To solve this problem, two measures have been taken in the new ultrasound urine sensor. Once a measurement is made, cautious discrimination of the raw data is first conducted and the reliable ones are selected. A simple average method is then employed to yield a smoothed measurement index, $P D$. It is found that the ruggedness of the measurement signals has been suppressed, substantially.

Up to now, a number of prediction models have been proposed and employed to estimate the urine accumulation in the bladder and they can be generally divided into two broad categories: mechanical and spatial models. The former infers the urine accumulation in the bladder based solely on the information from the echoes obtained. It depends heavily upon the proper interpretation of the underlying relationship between the shape of the bladder and the characteristics of the echoes received. Although it is likely to lead to a relatively simple design, the measurement accuracy sometimes appears to be difficult to maintain $^{3)}$. The latter calculates the urine accumulation in the bladder based on the detailed shape of the bladder ${ }^{8), 12), 13)}$. Although it seems to be more reasonable than the former from a mathematical point of view, very complicated designs are often unavoidable due to the necessity of 
determining exactly the spatial structure of the bladder. With reference to the two categories of prediction models above, a simple one is developed and used in the new ultrasound urine sensor. The urine accumulation in the bladder can be calculated by the following equation.

$$
V=c_{0} P D+c_{1}
$$

where $c_{0}$ and $c_{1}$ are the constants that specify the underlying characteristics of each user. They can be easily estimated with the following least-squares regression method.

$$
\begin{aligned}
& C_{0}=\frac{\sum_{j=1}^{N}\left(P D_{j}-P D_{a v}\right)\left(V_{j}-V_{a v}\right)}{\sum_{j=1}^{N}\left(P D_{j}-P D_{a v}\right)^{2}} \\
& c_{1}=V_{a v}-c_{0} P D_{a v} \\
& P D_{a v}=\frac{\sum_{j=1}^{N} P D_{j}}{N} \\
& V_{a v}=\frac{\sum_{j=1}^{N} V_{j}}{N}
\end{aligned}
$$

Therefore, prior to the use of the new ultrasonic urine sensor, the parameter, $c_{0}, c_{1}$, should be determined carefully by a certain number of practical measurements $(N=$ $12 \sim 20$ ). After determination of these parameters, they can be used for quite a long time. Moreover, it is reasonable to interpret Eq. (2) as a formulation to calculate the volume of the bladder based on the available information on its height and bottom area. It combines actually the advantages of both the mechanical $\left(P_{i}, i=1, \cdots, n\right)$ and the spatial $\left(D_{i}, i=1, \cdots, n\right)$ models, hence featuring relative simplicity and accuracy.

\section{Test Results and Analysis}

Clinical evaluations of the new ultrasonic urine sensor have already been carried out in a lot of medical facilities and fairly good results have been achieved so far ${ }^{14) ~ 17)}$. Here, the results of two experimental tests performed in the Takeshiba Engineering Incorporation and the National Institute of Advanced Industrial and Science Technology of Japan (AIST) are introduced in the sequel. Prior to each experimental test, full written consent has been received from all the persons recruited. During the measurements, they are all in a supine position.

In Fig. 5, a typical result of the experimental test performed in the Takeshiba Engineering Incorporation is illustrated. A person of 30 years was measured intensively with the new ultrasonic urine sensor in 8 days and totally 29 times of urine voiding was documented. It is noted that the data obtained situate quite closely to the diagonal line of $45^{\circ}$, thus demonstrating a fairly good indication of the

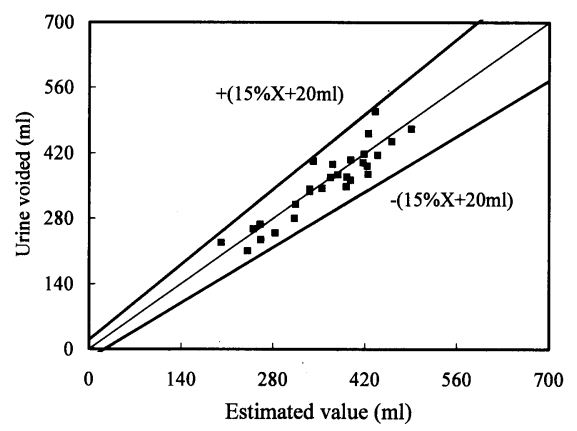

Fig. 5 Relationship between the estimated bladder volume and the amount of urine voided (in the Takeshiba Engineering Incorporation)

Table 1 Analysis of the test results in the Takeshiba Engineering Incorporation

\begin{tabular}{c|c|c|c}
\hline \hline $\begin{array}{c}\text { Test results } \\
\text { in the Takeshiba } \\
\text { Engineering Incorporation }\end{array}$ & $\begin{array}{c}\text { No. of } \\
\text { voiding }\end{array}$ & Percentage & $\begin{array}{c}\text { Average } \\
\text { error }\end{array}$ \\
\hline Relative error within 5\% & 12 & $41.4 \%$ & $2.23 \%$ \\
\hline Relative error within 10\% & 21 & $72.4 \%$ & $4.34 \%$ \\
\hline Relative error within 15\% & 28 & $96.5 \%$ & $6.38 \%$ \\
\hline Relative error within 20\% & 29 & $100 \%$ & $6.72 \%$ \\
\hline
\end{tabular}

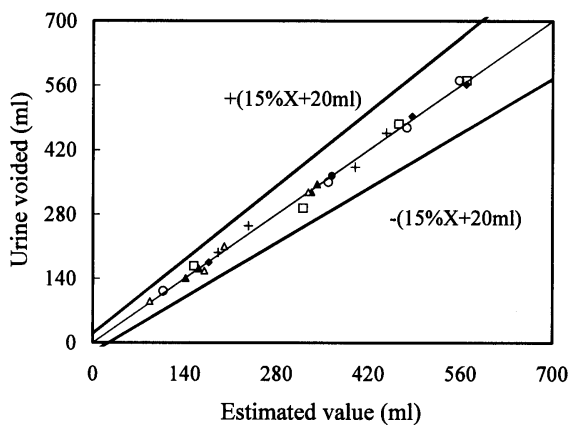

Fig. 6 Relationship between the estimated bladder volume and the amount of urine voided (in the AIST)

urine accumulation in the bladder by the new ultrasonic urine sensor. All the measurement values fall into an acceptable region of $\pm(15 \%+20 \mathrm{ml})$ and Table 1 lists a more detailed analysis of the experimental test.

Fig. 6 shows the results of experimental test conducted in the AIST. 8 persons (including 4 men and 4 women) in normal health were recruited to take the test and totally 9 times of measurements were conducted. The span of the experimental measurement ranged from 6 to 7 hours for each person. However, because of the specific characteristics of each person tested, the time to void their bladders was different. Again, it can be found that the new ultrasonic urine sensor can present a very satisfactory prediction of the urine accumulation in the bladder, with a relative measurement error less than $\pm(15 \%+20 \mathrm{ml})$. An indepth analysis of the test results can be found in Table 2 . 
Table 2 Analysis of the test results in the AIST

\begin{tabular}{c|c|c|c}
\hline \hline Test results in the AIST & $\begin{array}{c}\text { No. of } \\
\text { voiding }\end{array}$ & Percentage & $\begin{array}{c}\text { Average } \\
\text { error }\end{array}$ \\
\hline Relative error within $5 \%$ & 19 & $79 \%$ & $1.90 \%$ \\
\hline Relative error within $10 \%$ & 24 & $100 \%$ & $3.15 \%$ \\
\hline
\end{tabular}

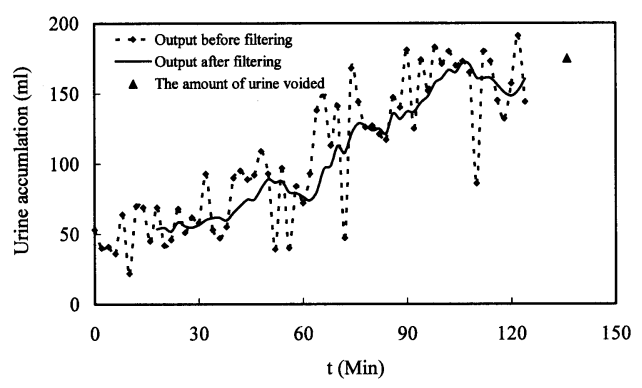

Fig. 7 Performance of the new ultrasonic urine sensor when it is used as a real time monitor

In Fig. 7, the performance of the new ultrasonic urine sensor is examined when it is used as a real time monitor of the urine accumulation in the bladder. As can be seen, a very reasonable prediction has been achieved on the amount of urine accumulated in the bladder. In particular, the predicted value is in good agreement with the amount of urine voided at the time instant of $2.25 \mathrm{hr}$, with a relative measurement error of only $-8.57 \%$. Furthermore, the effect of employing a simple average filter in the new ultrasonic urine sensor can also be easily identified in this figure. It is noted that the output ruggedness caused by the ultrasound beams has been alleviated substantially, thereby improving the accuracy and robustness of the new ultrasonic urine sensor, substantially.

One issue that is worth mentioning here is how to iden tify the place to fix the transducer on the abdomen. It is a critical problem in using the new ultrasonic urine sensor, which can have a significant effect on the measurement of urine accumulation in the bladder. Based on the MRI analysis, it is readily understood that the pubis is a good indication of the place of the human bladder. To allow the ultrasonic signals to be reflected from the bladder, the place to fix the transducer should be just above the pubis. After intensive experiment test, it has been proved to be the best place for the new ultrasonic urine sensor. This rule is actually developed in the preliminary clinical test and summarized later as a general stipulation. It is no doubt that the angle to place the transducer can have a certain effect on the measurement accuracy. However, to arrange it along the cephalic direction will not give any serious influences. During the practical measurements, the transducer is fixed actually by a tape and/or a belt, no particular problems have been found so far.

\section{Discussions}

One potential advantage of the new ultrasonic urine sensor is that it can offer a possibility to cope with the inherent nonlinearity of the bladder expansion process. If the degree of the inherent nonlinearity appears to be very severe for some users due to some physiological reasons (for instance, as shown in the third subgroup of persons tested in section 3 ), then it is impossible to get a satisfactory measurement with the linear model embedded (c.f., Eq. 2). To overcome this difficulty, a multi-model representation of the bladder expansion process looks very attractive in this situation. Re-examining Fig. 1, one may figure out that the bladder takes approximately a shape like an oblique cone when the amount of urine attained is small. With the accumulation of urine, it turns approximately into a cylinder and finally into a cylinder plus a semi-sphere. These realities provide us with definite evidence for the adoption of multi-models in the prediction of the urine accumulation in the bladder. In fact, the relative magnitudes of the measurement index, $P_{i} D_{i}(i=\mathrm{a}$, $\mathrm{b}, \mathrm{c}, \mathrm{d})$ can provide a very good indication of the variations in the shape of the bladder. Four linear models can be developed to infer the volume of the bladder in different ranges. It should be indicated here that multi-model representation of the bladder expansion process is likely to work as an effective way to improve the measurement accuracy of the new ultrasonic urine sensor. It may have special significance to the measurement of the bladder when the amount of urine retained is small (i.e., less than $100 \mathrm{ml}$ ), because the measurement accuracy appears to be more difficult to maintain than in the other situations. Currently, the work on the development and evaluation of the multi-model representation is underway. Preliminary results show that it can really offer a higher accurate measurement than Eq. (2) and the detailed result will be reported in our future paper.

\section{Potential Applications of the New Ultrasonic Urine Sensor}

The new ultrasonic urine sensor has a large potential to be widely used as a medical diagnostic apparatus in all kinds of hospitals, clinical treatment centers, nursing stations and individual families. With the guidance of the new ultrasonic urine sensor, the nursing care could become considerably easier than before for those patients who have been annoyed by urinary incontinence. In the mean time, the possibilities of involving new diseases can also be reduced substantially, including, for instance, pyelitis by 
the over-distention of the bladder, the infection by the adoption of indwelling catheters and so forth. Furthermore, the new ultrasonic urine sensor can even play an important role in assisting those older patients with prostatic hyperplasia. Because the residual of urine retained in the bladder can gradually lead to bladder dysfunction, checking the condition of the bladders is therefore necessary at each time after the patients have just voided their bladders. The information thus obtained can ease the anxiety of the patients and is of great assistance to the doctors as well.

One important way of using the new ultrasonic urine sensor is to train various patients with urinary incontinence. For instance, the incomplete control of urine discharging at night can frequently happen to children and even some adults. In order to overcome this problem, the patients must be trained intensively to know gradually the sensation from the bladder and thus understand the necessity and/or the proper time to void it. The new ultrasonic urine sensor can work effectively as a real time monitor for the bladder in this situation. The information about the distention of the bladder can be alerted to the patients and help them to know when the bladders should be voided. Another example goes to those patients suffering from the injury of their spinal cords caused by either accidents or diseases. They have to void their bladders through indwelling catheters due to the inability to do it themselves. However, the method has a serious side-effect because the bladders may gradually lose their function. With the help of the new ultrasonic urine sensor, the patients can be liberated from the stress of urinary incontinence as well as the limitation of water drinking. This kind of examples is now frequently reported by the nursing stations in Japan. Moreover, the new ultrasonic urine sensor is now working as a training facility in some nursing schools in Japan. Students can now understand more comprehensively than before the characteristics of urine accumulation in the human bladder.

The new ultrasonic urine sensor can also play an important role in the systematic administration of a nursing station. To provide satisfactory service to various patients with urinary incontinence, it appears to be necessary that the nursing personnel should plan their activities properly and efficiently. With the help of the new ultrasonic urine sensor, they can understand more accurately than before the characteristics of each patient and make deliberately the time schedule for serving the patient, thereby improving the quality of nursing service and reducing the intensity of work simultaneously. Moreover, the new ultrasonic urine sensor can also be connected to a computer system so that the history data measured can be stored and processed by the computer system. The socalled computer administrative system (Fig. 8) could help doctors substantially to gather and know the detailed information about each patient. When it is necessary to help a patient to void his bladder, the ultrasonic urine sensor based computer system can give alert signals and remind the nursing personnel of taking necessary actions. Currently, one such computer administrative system has already been developed and under practical tests now in some hospitals, clinical treatment centers and nursing stations in Japan.

The new ultrasonic urine sensor was patented in $2004^{18)}$ and permission was given on its medical applications by the Ministry of Health, Labor and Welfare of Japan in September of 2004 (No.21600BZZ00466000). The new ultrasonic urine sensor is already commercialized (Yuririn USH-052) and now available on the market ${ }^{19)}$. As shown in Fig. 9a, the new ultrasonic urine sensor can now be made so compacted in volume $(0.231)$ and light in weight $(210 \mathrm{~g})$, thereby making it very easy to be fixed on the low abdomens of various patients with urinary incontinence (Fig. 9b). Furthermore, the new ultrasonic urine sensor

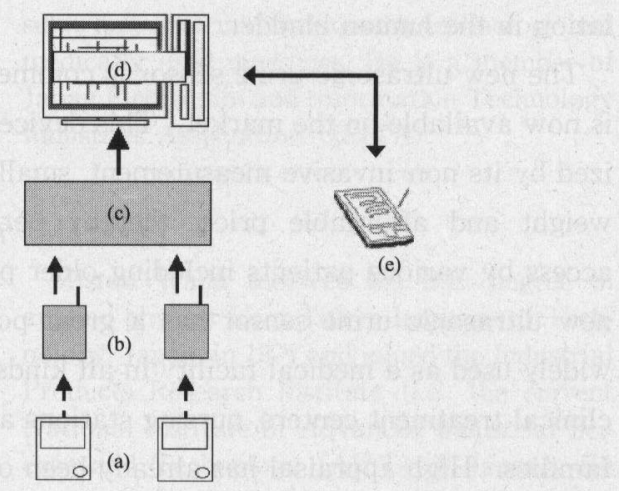

Fig. 8 Schematic of a computer administrative system for a nursing station. (a) ultrasonic urine sensor; (b) interface; (c) signal relay; (d) computer; (e) portable console

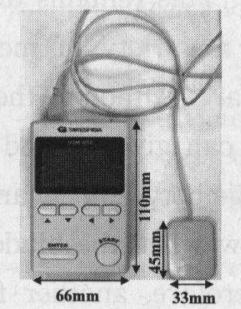

(a)

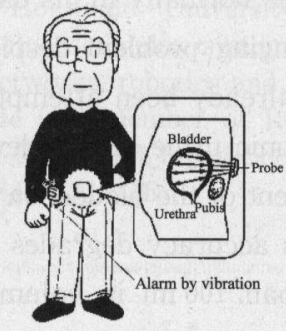

(b)
Fig.9 The new ultrasonic urine sensor commercialized and the simple way to use it 
has already been made so cheap that it can be afforded by various patients.

\section{Conclusions and Future Work}

A new ultrasonic urine sensor was developed for measuring the urine accumulation in human bladders. Based on the intensive observations on the bladder expansion process by means of a magnetic resonance imager, it was found that the bottom part of the bladder showed very small variations under the accumulation of urine. The primary changes in shape occurred actually at the top part of the bladder and they could simply be measured with the application of ultrasound beams. These observations help to lay a simple basis for the development of a new ultrasonic urine sensor. To evaluate the feasibility of the measurement principle, a clinical test was conducted in the Spinal Injuries Center and a strong monotonic tendency was identified between the bladder volumes and their predictions. Design of the new ultrasonic urine sensor was then conducted and further experimental tests were carried out with the developed apparatus in the Takeshiba Engineering Incorporation and the National Institute of Advanced Industrial and Science Technology of Japan. It was confirmed that the new ultrasonic urine sensor could really display a very good indication of the urine accumulation in the human bladder.

The new ultrasonic urine sensor is commercialized and is now available on the market. This device is characterized by its non-invasive measurement, small volume, low weight and affordable price, thereby permitting easy access by various patients including older patients. The new ultrasonic urine sensor has a great potential to be widely used as a medical facility in all kinds of hospitals, clinical treatment centers, nursing stations and individual families. High appraisal has already been obtained from a great number of users.

One work that is worthwhile to do in the near future is the extension of the new ultrasonic urine sensor to measure the accumulation of urine in the bladder when its user behaves normally in his daily life. This remains to be a challenging problem despite that a variety of methods have already been attempted so far. Although the new ultrasonic urine sensor developed can give a good measurement of the bladder in the range between 100 and 900 $\mathrm{ml}$, its accuracy degrades somehow when the bladder is less than $100 \mathrm{ml}$ in volume. Therefore, another future work is to gain improvement in the measurement accuracy in the low volume region $(V \leq 100 \mathrm{ml})$. Furthermore, when the nonlinearity of the bladder expansion process appears to be severe for some patients, it is necessary to devise special prediction models to treat these particular cases. A multi-model representation might be a candidate method for overcoming this difficulty and it is now under investigation.

\section{Acknowledgment}

The authors are grateful to Dr. N. Sekido of the Institute of Clinical Medicine of Tsukuba University and Dr. E. Iwatsubo of the Consultation Office for Urinary Care of Kitakyushu Hospital (previously in the Spinal Injuries Center of Japan) for their persistent support during the development and clinical tests of the new ultrasonic urine sensor.

\section{References}

1) J.F. Pedersen, R. J. Bartrum and C. Grytter: Residual Urine Determination by Ultrasonic Scanning, Am. J. Roentgenol., 125, 474/478 (1975)

2) G. K. McLean and S. L. Edell : Determination of Bladder Volumes by Gray Scale Ultrasonograph, Radiology, 128, 181/182 (1978)

3) J. A. Companion, T.S. Heyman, T. N. Blalock, B. A. Mineo and A. R. Cavalier: Apparatus for Measuring the Volume of Urine in the Human Bladder, US Patent No. 929869 (1986)

4) G. M. Coombes and R. J. Millard: The Accuracy of Portable Ultrasound Scanning in the Measurement of Residual Urine Volume, The J. Urology, 152, 2083/2085 (1994)

5) H. Oh-oka and R. Nose: Efficacy and Problems of Bladder Volume Measurement using Portable Three Dimensional Ultrasound Scanning Device-In Particular, on Measuring Bladder Volume Lower than $100 \mathrm{ml}, J$. Urology Japan, 96 601/609 (2005)

6) H., Shinbo, S. Kageyama, T. Tsukada, K. Suzuki, K. Fujita and T. Nakanishi: The Value and Problems of Portable Ultrasound Scanner for the Determination of Residual Urine Volume, Rinsho Hinyokika, 53, 999/1002 (1999)

7) Y., Kato, Y. Sue, H. Fuji, A. Numuta, N. Taniguchi, S. Kaneko, S. Yachiku, M. Mizunaga and M. Miyata: The Accuracy of Portable Instrument for Ultrasound Bladder Capacity Measurement (BVI 5000), Rinsho Hinyokika, 53, 459/462 (2000)

8) D. Ganguly, T. L. Robert and R.F. McConaghy: System for Estimating Bladder Volume, US Patent No. 5964710 (1999)

9) Y. Higashi and T. Hirata: Atlas of Abdominal Ultrasonography, Nanzando Company Limited, Tokyo, Japan (1982)

10) Y. Higashi and H. Matsumoto: Approaches for Ultrasonic Diagnosis, Igaku-Shoin Ltd., Tokyo, Japan (1989)

11) K. Chihara: Ultrasonic Image Processing, Corona Publishing Corporation Limited, Tokyo, Japan (2001)

12) B. T. Haylen, M. I. Frazer, J. R. Sutherst and C. R. West : Transvaginal Ultrasound in the Assessment of Bladder Volumes in Women, British J. Urology, 63, 149/151 (1989)

13) N. Simforoosh, F. Dadkhah, S. Y. Hosseini, M. A. Asgari, A. Nasseri and M. R. Safarinejad: Accuracy of Residual Urine Measurement in Man: Comparison between RealTime Ultrasonography and Catheterization, The J. Urology, 158, 59/61 (1997) 
14) H. Kodama, I. Hieda, Y. Kuchinomachi, H. Takeichi and H. Matsumoto: Non-Invasive Ultrasonic Urine Sensor for Ambulatory Patient Support, Methods of Information in Medicine, 33, 97/100 (1994)

15) H. Kodama, Y. Kuchinomachi, I. Hieda, H. Yoshimura, K. Mizuno and T. Nishimura: New Sensor for Prevention of Urinary Incontinence, Proceedings of the 4th Asia-Pacific Conference on Medical \& Biological Engineering, 309/309 (1999)

16) H. Kodama, H. Yoshimura, J. Sugawara, Y. Nagata, E. Iwatsubo and K. Matsuo: Development of a Wearable Ultrasonic Sensor for Bladder Dysfunction, International Ergonomics Association XVth Triennial Congress, Seoul, Korea (2003)

17) H. Kodama, J. Yu, E. Iwatsubo, Y. Kuchinomachi, H. Yoshimura and N. Kuchitsu: An Ultrasonic Urine Sensor for Aged People, Promoting Independence for Older Persons with Disabilities, 18, 223/230 (2006)

18) H. Kodama : An Ultrasonic Urine Sensor, Japan Patent No. 105737 (2004)

19) Website of Takeshiba Engineering Incorporation, http:// www.yuririn.jp/

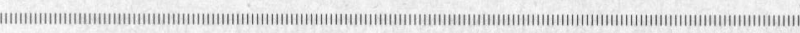

\section{Hiroyuki Kodama}

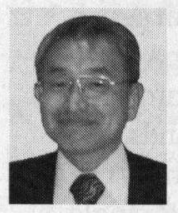

Hiroyuki Kodama received his B.S. and M.S degrees in theoretical physics from University of Tokyo in 1968 and 1971, respectively. He joined the Industrial Products Research Institute (i.e., the current National Institute of Advanced Industrial Science and Technology (AIST) of Japan). His research fields include human factors engineering, medical and biological engineering and assistive technology. He is a member of Japanese Society for Medical and Biological Engineering (JSMBE), Japan Ergonomics Society (JES), and Japanese Society of Biofeedback Research (JSBR).

\section{Kejin Huang}

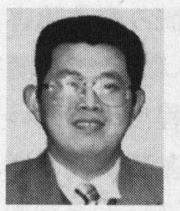

Kejin Huang received his B.E., M.E., and Ph. D. degrees, all in control theory and control engineering, from Zhejiang University, Hanzhou, Zhejiang, P. R. China, in 1986, 1989 and 1992, respectively. He worked in the Institute of Industrial Process Control of Zhejiang University firstly as an assistant professor and then as an associate professor from 1992 to 1995. Since then, he has been a research fellow in the National Institute of Advanced Industrial Science and Technology (AIST) of Japan. His current research fields include process synthesis and design, process integration and intensification, process modeling, control and optimization, signal detection and processing, etc.

\section{Jing Yu}

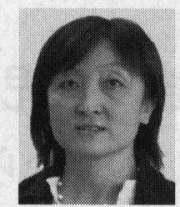

Jing $\mathrm{Yu}$ graduated from Nanjing Navy Medical College, Nanjing, Jinagsu, P. R. China, in 1986. She then worked in the $409 \mathrm{Navy}$ Hospital, Qingdao, Shangdong, P. R. China, until 1997. Since 2001, she has worked as a research assistant in the National Institute of Advanced Industrial Science and Technology (AIST) of Japan.

\section{Yasuo KuchinomachI}

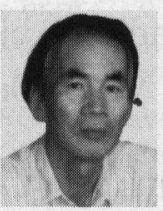

Yasuo Kuchinomachi received his B.S. and M.S degrees in psychology from University of Kyoto and joined the Industrial Products Research Institute (i.e., the current National Institute of Advanced Industrial Science and Technology (AIST) of Japan) in 1972. From 2004, he is a professor of the Shizuoka University of Welfare. His research fields include human factors engineering, geron-psychology, and assistive technology. He is a member of the Japanese Psychological Association and the Japan Ergonomics Society.

\section{Hisashi Yoshimura}

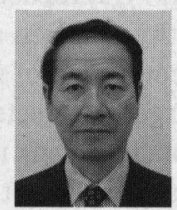

Hisashi Yoshimura graduated from Tokai University in 1972, studying the molecular movement in the surface of metal. He is now the head of research and development department in the Takeshiba Engineering Inc. His main research fields are development of ultrasonic non-destructive diagnosis technology and medically-used machines. He is a member of Japan Electronics and Information Technology Industries Association (JEITA).

\section{Mitsuo WadA (Member)}

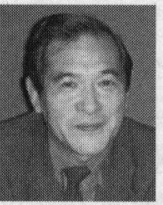

Mistuo Wada received his B.E. degree in applied physics from Tokyo Institute of Technology, Japan, in 1971 and joined the Industrial Products Research Institute (i.e., the current National Institute of Advanced Industrial Science and Technology (AIST) of Japan). He received the Ph.D. degree in mathematical engineering from University of Tokyo in 1987. From 1995 to 2003, he was a professor of the graduate school of engineering of Hokkaido University, and since 2003 he is Professor of the graduate school of Information Science and Technology of Hokkaido University. His research fields include complex systems engineering, neural networks, robotics and human skill interface. He is a member of Robotics Society of Japan (RSJ) and Japan Society of Mechanical Engineers (JSME). 